\title{
Accelerated Assessment of Critical Infrastructure in Aiding Recovery Efforts During Natural and Human-made Disaster
}

\author{
Gautam Thakur, Kelly Sims, Chantelle Rittmaier, Joseph Bentley, Debraj De, Junchuan Fan \\ Tao Liu, Rachel Palumbo, Jesse McGaha, Phil Nugent \\ Bryan Eaton, Jordan Burdette, Tyler Sheldon, Kevin Sparks \\ \{thakurg,simskm,rittmaiercm,bentleyjd,ded1,fanj\}@ornl.gov,taoliu@mtu.edu \\ \{palumborl,mcgahajr,nugentpj,burdetteja,sheldondt,sparkska\}@ornl.gov \\ Human Dynamics Section, Geospatial Science and Human Security Division, Oak Ridge National Laboratory, USA
}

\begin{abstract}
Relief and recovery from disasters (both natural and human-made) require a coordinated approach across several federal and state government agencies. In order to achieve optimal resource allocation and deployment of first responders, accurate and timely assessment of the impact and extent of destruction are the cornerstones to any recovery effort. Ideally, this knowledge should be gathered and shared within the first 0-24 hours (termed as "Acute Phase" by the U.S. CDC guideline) for informed decision-making. But achieving this poses significant challenges for the data collection and data harmonization processes, particularly when voluminous data are being generated from diverse and distributed sources during the disaster responses. To this end, this work developed a scalable and efficient workflow to dynamically collect and harmonize crowd-sourced geographic multi-modal data, and then assess critical infrastructure (CI) damaged during disaster events. We demonstrate the application of our framework with two real-world experiences in addressing post-disaster recovery efforts - for the Bahamas (Natural - due to Hurricane Dorian, 2019) and Beirut (Human-made - due to explosion caused by the ammonium nitrate stored in a warehouse, 2020). We have illustrated that a coordinated effort is needed for planning as well as for execution to achieve informed decision making.
\end{abstract}

\section{CCS CONCEPTS}

- Information systems $\rightarrow$ Geographic information systems; Decision support systems; • Computer systems organization $\rightarrow$ Data flow architectures; $\bullet$ Computing methodologies $\rightarrow$ Machine learning.

\section{KEYWORDS}

Spatial data mining and knowledge discovery, geographic information retrieval, disaster response, geographic information system, assessment of critical infrastructure, damage assessment, data curation and management, data reliability and quality, machine learning

This work is licensed under a Creative Commons Attribution-

NonCommercial-ShareAlike International 4.0 License

SIGSPATIAL '21, November 2-5, 2021, Beijing, China

(C) 2021 Copyright held by the owner/author(s).

ACM ISBN 978-1-4503-8664-7/21/11.

https://doi.org/10.1145/3474717.3483947

\section{ACM Reference Format:}

Gautam Thakur, Kelly Sims, Chantelle Rittmaier, Joseph Bentley, Debraj De, Junchuan Fan, Tao Liu, Rachel Palumbo, Jesse McGaha, Phil Nugent, and Bryan Eaton, Jordan Burdette, Tyler Sheldon, Kevin Sparks. 2021. Accelerated Assessment of Critical Infrastructure in Aiding Recovery Efforts During Natural and Human-made Disaster. In 29th International Conference on Advances in Geographic Information Systems (SIGSPATIAL '21), November 2-5, 2021, Beijing, China. ACM, New York, NY, USA, 12 pages. https://doi.org/10.1145/3474717.3483947

\section{INTRODUCTION}

Accelerated assessment of damages to Critical Infrastructure or CI (such as schools, hospitals, roads and bridges and other transportation components, energy, food, telecommunication, etc.) is cornerstone to the post-disaster planning and mitigation efforts. Such planning efforts include creation of temporary shelters, identification of evacuation zones, and optimal placement of first-responders, among the others. A coordinated approach is needed that includes multitude of activities: a) Top-down or high-level damage estimation from remote sensing data, b) human in loop validation and verification of CIs that fall in the path of disaster from the previous step, c) Bottoms-up or higher-order feature extraction from spatially-explicit ground-level imagery (also serves as a second line of evidence), and d) information extraction along with information quality assurance from crowd-sourced data (social media, news, blogs, etc.). Besides, robust scalable algorithms and data-intensive computing infrastructure are essential for creating a comprehensive picture of the immediate event aftermath situation, and delivering it within 24 hours of post-disaster (called the "Acute Phase" by the U.S. CDC guideline [2]), for maximal effectiveness.

Unfortunately, current approaches and ERM (emergency response management) pipeline in practice are limited in their capacity to utilize the full values of multitude of analytics, modeling, algorithmic and computing solutions [8, 9, 18, 20]. For example, the work in [20] has found through conversations and collaborations with first responders that there is limited use of optimized dispatch and informed decision-making after the incident occurrence. This is happening in real-world practice for two reasons [20]: (i) response to incidents follow a greedy strategy and geographically closest approach, for instantaneous dispatch effort after a report is received (despite the fact that optimizing dispatch can minimize response times in the long run); (ii) it has been hard to judge the severity of an incident from a service call. In 2017 hurricane season, afteraction report [3] by FEMA (the Federal Emergency Management Agency) it is stated that ".. FEMA and supporting federal agencies 


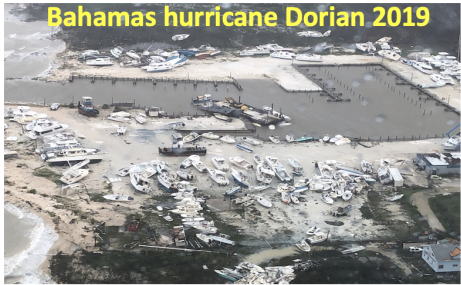

Beirut explosion at port 2020

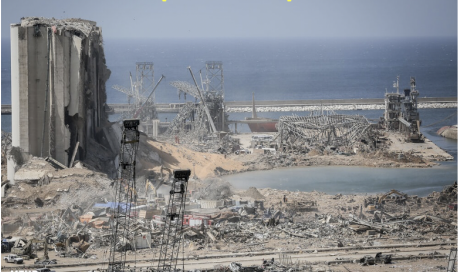

(a) Damage due to natural and humanmade disaster events.

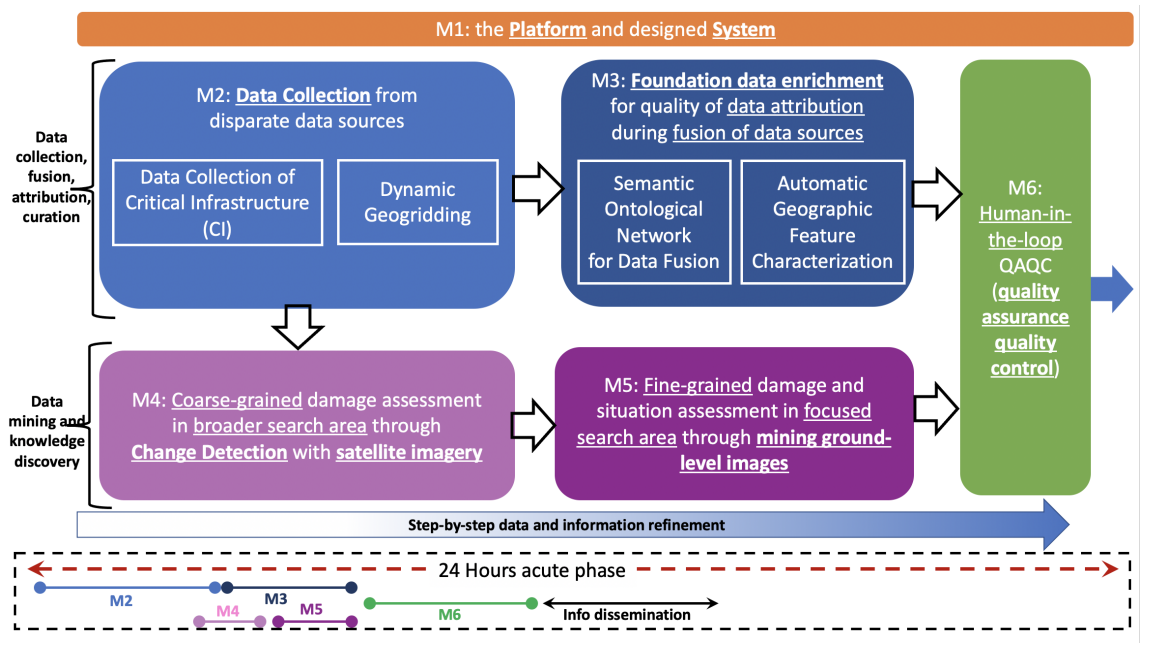

(b) Overall modules of our designed designed system for accelerated assessment of CIs (Critical Infrastructure) in immediate post-disaster situation.

Figure 1: Figure (a) illustrates: (i) damage due to Bahamas hurricane Dorian 2019 (natural disaster event); and (ii) damage due to Beirut explosion 2020 (human-made disaster). Figure (b) describes overall modules and data workflow in our system solution.

struggled to gain situational awareness and assess the status of critical infrastructure .." and ".. diminished situational awareness continued through the first 72 hours after landfall ... This led to the development of FEMA's 2018-2022 Strategic plan [4]. Currently, it takes a long-time to generate effective insights, while also losing the critical time window for an optimal mitigation strategy.

Our work is highly motivated by FEMA's 2018-2022 strategic plan's [4] "Strategic Goal 3: Reduce the complexity of FEMA ... We must innovate and leverage new technology to reduce complexity, increase efficiency, and improve outcomes". Overall modules (M1M6) and data workflow of our designed system are illustrated in Figure $1 \mathrm{~b}$. The timeline shown below in the figure also illustrates the overall processing time that the modules took for the large-scale CIs assessment, in events scenarios shown in Figure 1a. Although the timeline didn't consider potential bottleneck in obtaining satellite images, due to various vendors and protocols. Now, our work has designed both: (I) multi-level assessment of geospatially distributed critical infrastructure (CI) through multi-modal data sources and innovative algorithmic strategies; and (II) make the CI assessment process accelerated for timeliness and actionable values, through our designed and deployed data-intensive computing platform and algorithms. Among the algorithmic strategies, we have designed: (i) dynamic geo-gridding mechanism for more contextual focus on geo-spatially affected areas of concern; followed by (ii) satellite imagery based change detection (for high-level broader assessment) in identified geo-grids of concern; and then (iii) ground-level social media photos based complex information extraction (for more local and fine-grained assessment); and at the same time (iv) handling data heterogeneity and attribution dissimilarity in crowd-sourced geographic data through a semantic ontological network based data fusion. We have also (v) developed an end-to-end multi-modal data-intensive geospatial computing platform, based on lambda architecture [19], that's capable of ingesting disparate data sources (text, ground-level photo, remote sensing imagery, other crowdsourced data), and processing them in near real-time. Our platform also acts as a Data-as-a-Service (DAAS) for integration to thirdparty applications and services.

Our presented work integrates multiple research areas that include the following relevant fields: spatial data mining and knowledge discovery, geospatial architectures and middleware, spatial knowledge graphs, geographic information retrieval, spatial data quality and uncertainty, location-based services, geospatial computer vision applications, earth observation and photogrammetry. In this work, we have also discussed our collective experience in mapping damages to CIs during two disaster events of different kinds (one natural, another human-made) as shown in Figure 1a. First one is the natural disaster when hurricane Dorian struck the Abaco Islands as a Category 5 hurricane on September 1, 2019, and a day later hit Grand Bahama Island at the same category [25]. The second one is a human-made 2020 Beirut explosion when a large amount of ammonium nitrate stored at the port of the city of Beirut, the capital of Lebanon, exploded resulting in severe damages to human lives and infrastructure [23]. An informative media coverage of our work is available for interested readers at this location - https://www.ornl.gov/news/planetsense-stepping-when-disasterstrikes

\section{M1: PLATFORM}

Several web-based and interactive platforms have been built that provide support towards disaster mitigation; It is difficult to enlist all given there's a plethora of them, some includes $[6,7,10,12,21$, $29,30]$. Our work can be integrated with these platforms through third-party data-as-a-service. As shown in Figure-2, a Lambda architecture $[15,27]$ inspired real-time geospatial data-intensive computing platform is developed and operationalized to take advantage of both batch and stream-processing methods combining historic 


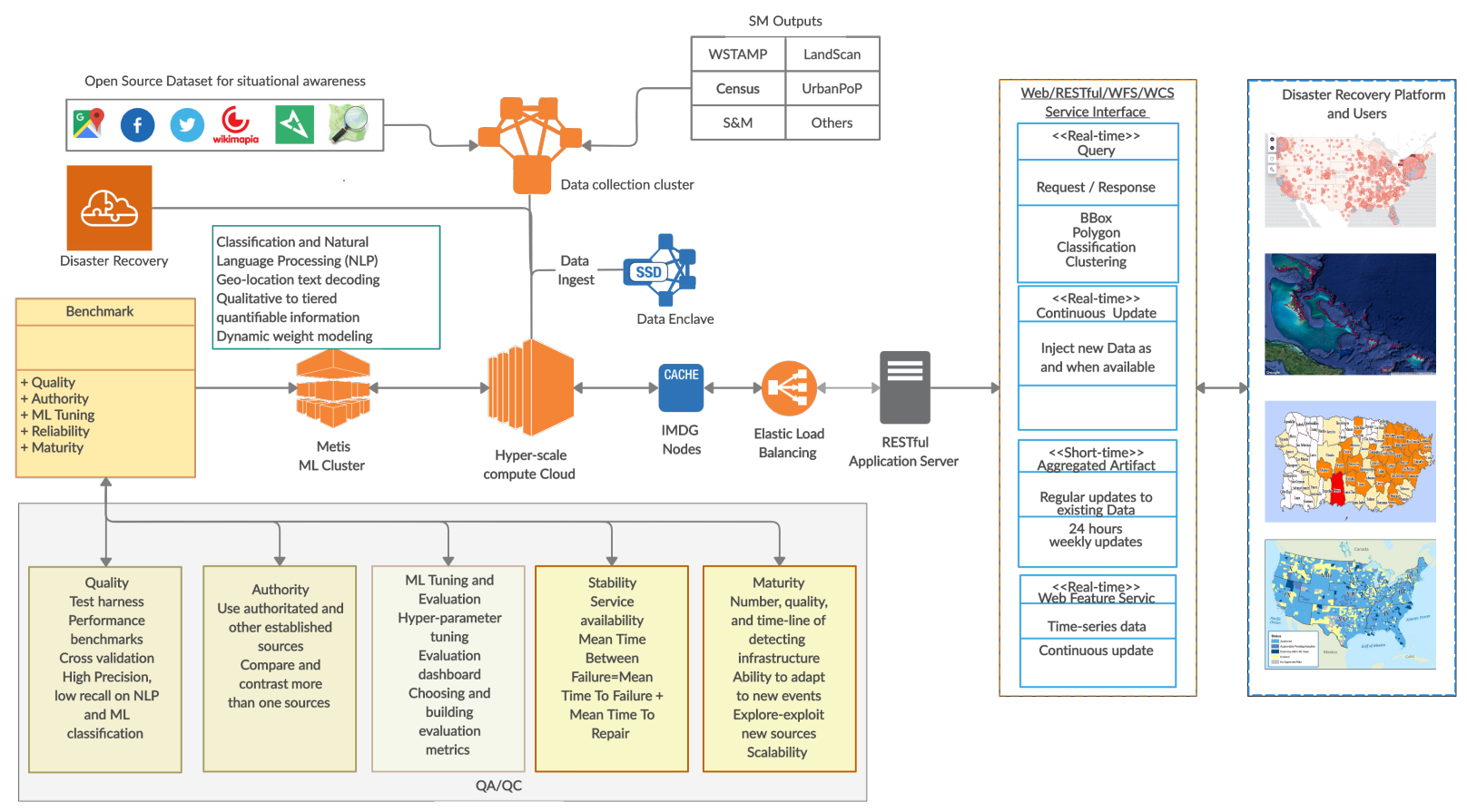

Figure 2: Our overall platform for data collection and data-intensive computation, for accelerated assessment of Critical Infrastructure (CI) for aiding recovery efforts during disaster situations.

and real-time streaming data for effective decision making. The main components of the platforms are:

- Data collection cluster: A set of 60 virtual machines are deployed across the planet and are responsible for sensing and collecting disaster-related information from over 30+ sources (social media, news, blogs, IoT, sensors, etc.). After initial processing, this data is pushed to hyper-scale compute cloud for storage and further analysis.

- Hyper-scale compute cloud (HSCC): This direct-attached storage (DAS) with 1000 CPU-core cluster and dual 20gpbs ethernet connection serves as a central repository for data processing, anomaly detection, feature extraction, classification, etc. in near real-time.

- Machine/Deep learning cluster: A GPU-cluster connected to HSCC is deployed with distributed deep and machine learning algorithms for feature extraction, classification, and data mining.

- Human-Machine-Teaming: In addition to using automated methods (such as feature extraction, scene classification), a human-in-the-loop quality assurance and quality control for verification and validation of volunteered geographic data allowing detailed understanding spatial patterns across landscapes, regions, cultures, etc. This process enables a trustworthy dataset of spatially explicit CIs for the whole world by ranking CIs at various confidence levels.

- IMDG nodes: An In-Memory-Distributed-Grid middle-ware is developed and deployed on HSCC to provide extremely high throughput, reduce latency, and improved fault-tolerance of geospatial data by keeping it in distributed memory (cache). We load several Terabytes of CI layers and spatially-explicit ground-level imagery of disaster into the memory for near real-time access. A performance benchmark is shown in Appendix-A.1

- Elastic load balancing: Deployed data and services accessed during disaster experience uneven workload. A load-balancer is installed at the gateway level to distribute the incoming traffic across multiple application nodes.

- Application server: The compiled list of CIs are available through RESTful services API for humanitarian and other emergency management agencies to access the data.

Next, we discuss our approach to rapidly scan AOIs (areas of interest) for possible CIs (critical infrastructure) and relevant crowdsourced and volunteered geographic information.

\section{M2: DATA COLLECTION FROM DISPARATE DATA SOURCES}

In this section we present our system's overall mechanism for rapid collection of relevant data from disparate data sources.

\subsection{Data Collection of Critical Infrastructure Entities (CIs)}

As was illustrated in Figure 1b, our system collects Critical Infrastructure (CI) related multi-modal raw data from various open data sources (social media, online geospatial news and other content sites, and other web-based data sources, satellite imagery). These 


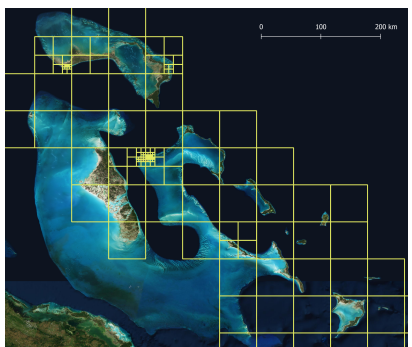

(a) Bahamas dynamic geogrid.

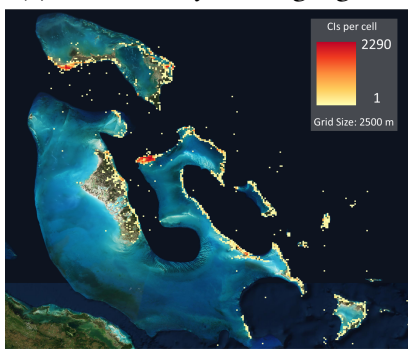

(c) CI distribution in Bahamas.

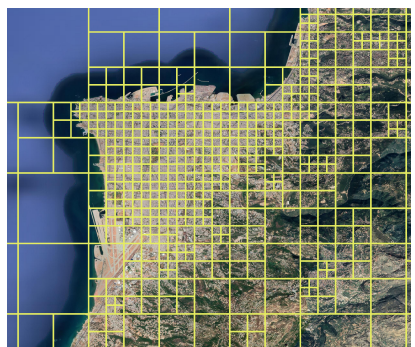

(b) Beirut dynamic geogrid.

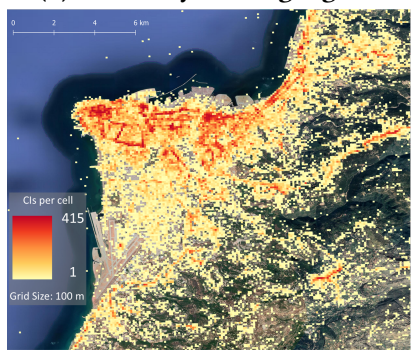

(d) CI distribution in Beirut.
Figure 3: Figures (a) and (b) are illustrating dynamic geo-grid computed for Bahamas and Beirut respectively. Figures (c) and (d) are illustrating identification of CIs (critical infrastructure) for Bahamas and Beirut respectively.

raw data are then curated and enriched, followed by standardization into certain schema based data layer. These standardized formatted data are then analyzed through machine learning and other algorithmic process, along with some human-in-the-loop operations for quality assurance. The final insights generated about assessment of CIs (along with collected and formatted datasets) are then made available as Data-as-a-Service layer.

Overall collected Critical Infrastructure (CI) data and their geographic locations are illustrated in Figure 3 (c) and (d). For The Bahamas hurricane 2019 event, there were total 23,674 CIs data collected, and 3,697 CIs were sampled through quality assurance process. On the other hand for Beirut explosion 2020 event, there were total 111,488 CIs data collected, and 1,989 CIs were sampled through quality assurance. For ground-level images or ground photos from social media, our system collected 265K images for Bahamas 2019 event, and $220 \mathrm{~K}$ images for Beirut 2020 event.

\subsection{Dynamic Geo-gridding}

In order to optimize the collection of geospatial data (CIs data in our case), it is a recommended practice to segment a given geographical region into smaller grid cells, for a more focused collection effort. ArcMap's fishnet [11], and other spatial indexing techniques that implement triangular mesh gridding are commonly used geoprocessing tools for this geo-grid segmentation. However, these tools are agnostic to the underlying topology and evenly segments areas into equally sized cells and/or triangular facets. Such approaches cannot skip unwanted regions (such as oceans) and their computational time is proportional to the desired cell size.
These challenges led us to develop a new approach for geo-grid segmentation that make use of underlying topology, such as population distribution, building footprint extractions, etc. The proposed method creates dynamically spaced geo-grids as a function of underlying topological data. Geo-grid algorithms divide a large polygon area into several smaller polygons, which are important for studying or executing a set of operations on underlying topological features of a map. In this work, we propose a novel dynamically spaced geogrid segmentation algorithm, that overcomes these challenges and provides a computationally optimal output for borderline cases of an uneven polygon. Our method uses an underlying topological feature of population distributions, from the LandScan Global 2016 dataset [5], for creating grids as a function of these weighted features. We benchmark our results against available algorithms and found that our approach improves geo-grid creation. Our proposed approach is more effective and time-efficient in harvesting CIs (critical infrastructure) data from a crowd-sourced platforms.

3.2.1 Spatial Analysis. We began by gridding the world in "0.6 $\mathrm{x}$ 0.6 " degree increments for two reasons. The first reason was to begin with a small enough grid size so that the largest possible search radius (from the center of a grid to the nearest edge) would be less than 50,000 meters. With that said, a 0.6 degree grid at the equator has a radius of roughly 33,300 meters. The rationale for not using a search radius just under $50,000 \mathrm{~m}$ (which would actually be a 0.9 degree grid at the equator), leads us to our second reasoning.

Our geohashing technique splits each grid into 4 equal parts, until the last grid becomes smaller than a LandScan Global [5] cell size (additional illustrations are in Appendix Section A.2 and Figure 11 ), which is $\sim 1 \mathrm{~km}$ at the equator. When a geohashed grid is smaller than a LandScan Global cell, we can no longer sum the population within the single grid, without missing at least one LandScan Global cell centroid. By using a 0.6 degree grid initially, we can geohash until the second to last geohashed grid is roughly $1 \%$ bigger than a LandScan Global population grid, allowing us to sum the population one last time. If the threshold is still not met, we can then geohash one final time to make the smallest request grid possible based on population. The population threshold for this study was 5,000 . Therefore, when a grid's underlying population sum was above 5,000 people, subdividing took place into 4 new quadrants recursively until the individual quadrant population threshold reach 5000 or below. The rest of the section discusses the algorithms aspect of our method.

3.2.2 Algorithm. The spatial analysis algorithm performs two important tasks that results in deciding whether to split the current grid cell in equal size small sub-grid cells, or move to next grid cell. This decision is made based on the population count in the current grid cell. If the population count is higher than the threshold, the algorithm (Algorithm 1) segments the grid cell. In this algorithm, first we calculate the extent of the current grid cell. An extent defines the geographic boundaries that contains a population data frame. These boundaries contain top, bottom, left, and right coordinates, which are the edges of the map extent. For the purpose of this work, we rely on fixed extent calculation of the cell. Later, we calculate the total grid cell population count from the raster centroid of this grid cell (Algorithm 2). The algorithm returns the value of population count, that the main algorithm uses to decide 
Function CalculateGeoHash (inputGrid, landScan_global, threshold)

Data: LandScan Global raster population layer, grid, and threshold values

Result: grid cells

/* Split the original grid in $0.6^{\circ}$ grid blocks. The radius from center is $33,000 \mathrm{mtrs}$ at the equator. API threshold 50,000 meters *1

intpuGrid $\longleftarrow$ calculate_fishnet (inputGrid, 0.6); overlay_landscan_global();

while true do

/* Traverse each grid block in sequential fashion *1 RunSpatialAnalysis (gridId, GeoPoint geoPoint [4], long population Count) ;

if populationCount $>5000$ then

Split the cell in to four equal parts; replace old cell with four new cells ; if current_grid $==\operatorname{len}$ (grid) then end $\operatorname{break}()$

end

else | continue; /* move to next grid cell */ end

end

end

Algorithm 1: Algorithm to calculate grids using LandScan Global

whether to segment or maintain the current extent of the cell. In this algorithm, parameters are passed as reference to calculate the values of grid cell extent and cell population count.

\section{M3: FOUNDATION DATA ENRICHMENT}

The user-generated volunteered geographic information (VGI) has long-standing issues with attribution and quality assurance [24],

Function RunSpatialAnalysis (gridId, GeoPoint geoPoint, long populationCount)

Data: LandScan Global raster population layer, grid, and threshold values

Result: grid cell extent, populationCount of the grid cell if cell! $=$ null then

/* Genrate coordinate values for top_left, top_right, bottom_left, bottom_right corners of the grid cell */ geoPoint $\longleftarrow$ calculate_boundaries_spatial_extent () ;

/* Calculate population count for this grid cell

populationCount $\longleftarrow$ extract_landscan_spatial_statistics(geoPoint) ; end

end

Algorithm 2: Algorithm to calculate grid cell extent \& population. for example, failing to appropriately classify map features, incomplete information about certain attributes (hours of operations), wrong geocoding. These issues are exacerbated when multiple data sources need to be fused together. Each data source usually comes with its unique attribution format, creating duplication of records about the same spatial feature. These issues pose severe challenges while creating reliable data products for first responders and local governments. In this section, we discuss the information infrastructure methodological framework that we have developed to tackle practical challenges that arise during timely response to disaster events.

\subsection{Semantic Ontological Network for Data Fusion}

Data heterogeneity and attribution dissimilarity across different data sources are common for non-authoritative crowd-sourced geographic data. This can present a major challenge when interlinking data sources for a rapid assessment of critical infrastructure in the event of a natural disaster. Moreover, a unified schema is needed for multi-source data in order to be used by data analysts at scale. A primary challenge is the heterogeneity of $\mathrm{CI}$ (critical Infrastructure) categorization schemes that exist across multiple data sources.

In [22], we had proposed a theoretical framework of semantic ontological network to handle heterogeneities of CI categorization across multiple data sources. We implemented it for this work and CI categories from each data source were interlinked using OpenStreetMap (OSM) tags, which are widely-utilized crowd-sourced mapping platform, particularly for humanitarian efforts. The OSM tagging schema consists of key:value pairs that identify the type of facility or location being mapped. Due to their diversity and structure, these tags were effectively matched to numerous and diverse data sets where $\mathrm{CI}$ categories vary not only in number, but also in semantic granularity (e.g., general categories such as grocery store, and hospital, coexist with more specific categories such as Italian Grocery and Trauma Care Hospital. The Semantic Ontology Network (SONET) is a graph database that we developed to be a permanent foundational data infrastructure and publicly available resource, and it is an integral part of our platform discussed earlier.

The SONET graph database contains links among 12,667 source categories from eight different CI data sources and is still growing. The schema illustrated in Figure 4 shows the hierarchical relationships among different node types. Each CI category from different data sources (i.e., source category) is mapped to a collection of OSM Tags based on its semantics. The OSM tags are the intermediate semantic bridge that connects CI categories from different data sources. A more specific example for the CI category Hospital is illustrated in Figure 5.

SONET plays a key role for the accelerated assessment of critical infrastructure (during all stages of the process) through providing a knowledge base foundation for the data fusion process of diverse data sources. Table 1 shows an example where sourceCategory for different types restaurants are mapped to the same osmCategory, therefore reducing the number of unique categories in the table from four to one across four different data sources. The application of standardized OSM tags and coarser semantic granularity (e.g., 


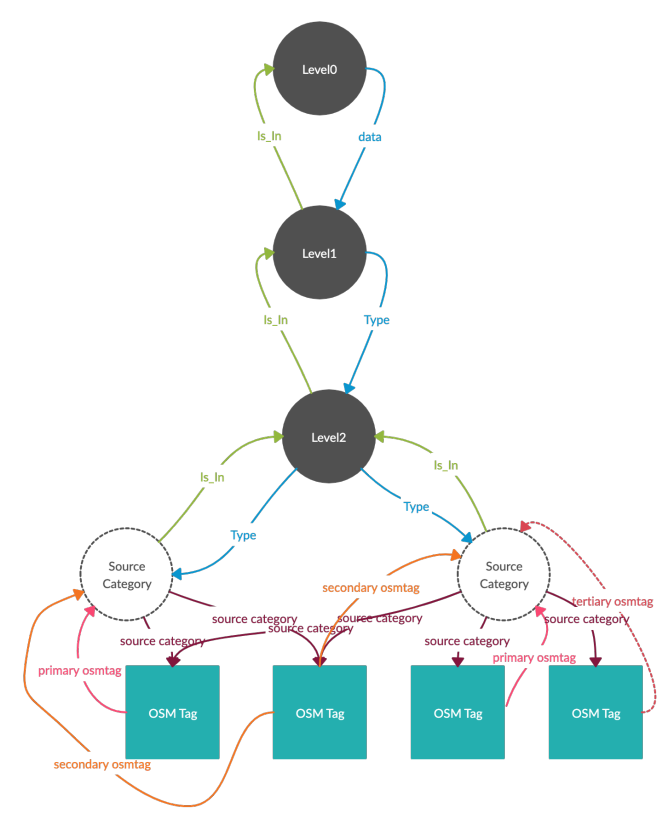

Figure 4: Example of SONET (Semantic Ontology Network) graph [22] capturing three different node types and the relationships between them. The filled in circular type nodes are the categoryLevel nodes that represent category groups at three different levels of semantic granularity, namely, categoryLevel0, categoryLevel1, and categoryLevel2. The hollow circular type nodes represent the sourceCategory nodes, capturing original categories of each data source. The box type nodes represent the osm Tag nodes (OSM: OpenStreetMap) that are used to generate the standardized osmCategory field. The links capture how different types of nodes are related to each other.

\section{Data Source sourceCategory osmCategory}

\begin{tabular}{lll}
\hline Facebook & Restaurant & amenity=restaurant;building=retail \\
Google & food & amenity=restaurant;building=retail \\
Wikimapia & eatery & amenity=restaurant;building=retail \\
HERE & Bistro & amenity=restaurant;building=retail
\end{tabular}

Table 1: Example of mappings between sourceCategory and osmCategory for different data sources.

Education and Healthcare) to each CI from a variety of data sources, expedites the process of identifying key infrastructure types for damage assessment (Table-2). Analysts can quickly filter CI data based on the unified CI categorization rather than needing in depth knowledge about the category schema and distribution of each data source. For example, across all data sources currently in SONET there are 52 unique categories for hospitals, but with the category standardization afforded by SONET, analysts only need to filter on one category amenity=hospital to identify all of the hospital type CIs. During the planning phase before a disaster, quickly being able to identify the location of CIs such as hospitals and schools can help decision makers identify and prepare areas that are critical to recovery efforts or are in potentially high danger zones.

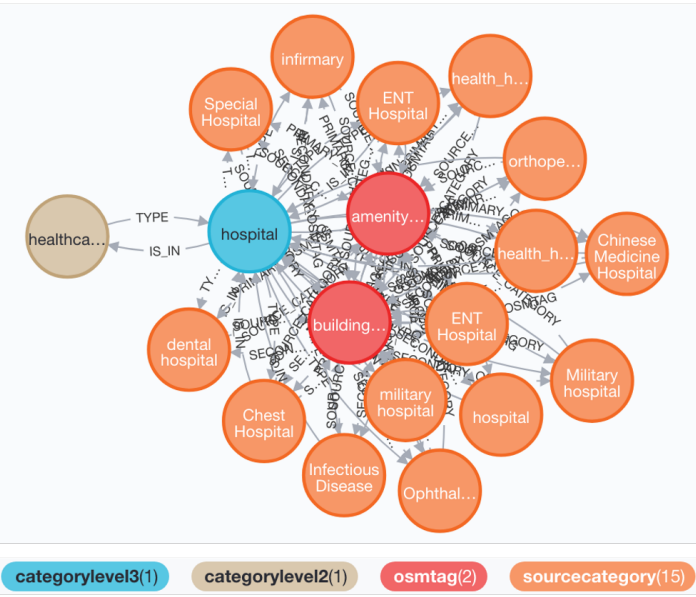

Figure 5: SONET (Semantic Ontology Network) subgraph for hospital type: source category names are linked with osmtTags amenity=hospital; building=hospital, and higher level categories as well.

\subsection{Automatic Geographic Feature Characterization}

Another challenge that is frequently encountered with VGI (volunteered geographic information) is missing or incorrect categorization of CI such as school, hospital, which can be impediments to accurate assessment of the status of the location as well as their livability. We have developed and deployed a module in our system that combines machine learning and natural language processing to work in tandem with the data collection process, in order to properly characterize and classify incorrectly categorized geographic features based on name and other attributes. Moreover, we also developed an auto-geocoding module for large amount of data that improves address and location mapping to the fifth decimal place. The fifth decimal place is worth up to 1.1 meter accuracy and therefore can distinguish a CI located on the road or on the building.

Feature and Category Mapping. Data collection from a large variety of sources naturally requires a large amount of data standardization to make the information usable. For each CI data collected, the name, category, and location are all translated to a more usable format. Of the source information, we observed that the category consistently was the most difficult to manage, given the large volume of CIs that are either poorly classified or not classified at all. With the rest of the information present, we have taken a step beyond translation into enhancement using machine learning models to attempt to generate a proper category based upon the name provided.

Methodology. The CIs data collected by our system have a wide variety of potential categories for classification, so generating training and test data (for machine learning based modeling) needed to be efficient and dynamic to allow for operational speeds that can keep pace with collection. To satisfy these goals, One-vs-All classification models were trained, separately for each category of interest (more details on rationale of this machine learning approach is provided in the Appendix in Section A.3). Thus separate data sets for each category of CIs, with sample data points numbered in at least the tens of thousands, were generated. Each such dataset consisted of concatenated data points, with one data point belonging 
Table 2: Non-Residential categoryLevel1 and categoryLevel2 Categories

\begin{tabular}{|c|c|c|c|c|c|c|}
\hline Retail & Institutions/Public Service & Commercial & Military & Agriculture & Entertainment & Transportation \\
\hline Food & Religious & Office Building & Facility & Outdoor Agriculture & Night Club & Rail \\
\hline Store & Education & Manufacturing & Base & Indoor Agriculture & Theater & Road \\
\hline Kiosk & Healthcare & Power Plant & Explosives & & Sports & Air \\
\hline Market & Public Service & Chemical Refining & Barracks & & Park & Sea \\
\hline Hospitality & Learning Facilities/Tourism & & & & Indoor Rec. & Warehouse \\
\hline
\end{tabular}

to the category of interest and another corresponding datapoint belonging to any other category. For example, given 40,000 "hospital" categorized points, the final "hospital" data set (for detecting "hospital" or "not hospital" in One-vs-All manner) would contain the 40,000 "hospital" data points and 40,000 data points that have any category other than "hospital" as the primary category. These data sets are then split into a training set and a test set with the test set containing a randomized selection of $20 \%$ of the set and the training data being the remaining $80 \%$ of the data set.

Moreover, groups of datasets are created for each source from which our system collects data, in order to ensure consistency in the meaning of a given category, relative to the source from which it was collected. Each of the groups of datasets are used to generate a set of binary models, that are then tested on the corresponding test dataset for the model generated. The binary model is used as the average performance of an SVM binary model and a Naïve Bayes binary model. The required number of points for a model to be utilized is then determined per source, based upon the average $F_{1}$ score from the results of the test datasets. This allows for the convenient expansion and contraction of possible categories for assignment, based upon the state of collected data.

\section{M4: CHANGE DETECTION USING REMOTE SENSING}

In this section we explain our work on satellite imagery based change detection, using the case of Bahamas 2019 hurricane event. Hurricane Dorian hit the Abaco Islands on September 1st 2019. To evaluate the extent of the impacted area caused by the hurricane Dorian, a pre-event remote sensing image collected on October 25th, 2018 and a post-event image obtained on September 5th, 2019 were analyzed in this study. The images are worldview-2 satellite images with 0.46 meter resolution and they consist of RGB and near-infrared channels.

The deep learning-based method proposed in [17, 26] was used to generate the change map in this study. This method utilizes two separate but identical CNN (convolutional neural network) modules to extract the features from pre- and post-event image patches and those features are concatenated before being fed into the classifier to generate the change type.

The training samples were automatically extracted from unchanged sampling area and changed sampling area. Two types of unchanged sampling area were generated from the study area covering the vegetation area and infrastructure area respectively. NDVI (normalized difference vegetation index [1]) was calculated for preand post-event images to extract the vegetation masks, which were then overlaid to each other to get the overlapping vegetation area as the unchanged vegetation sampling area. The SIFT (scale-invariant feature transform) feature detection method in computer vision was implemented to extract the unchanged keypoints, based on which the unchanged sampling area in the infrastructure area were generated. In addition, polygons were generated from pre-event water bodies as part of unchanged sampling areas given that the impact on water bodies brought about by tornado is not the focus of this study. The pre- and post-event images were visually inspected to create some polygons covering subareas where buildings were totally destroyed or pre- and post-event vegetation masks do not overlap, and those polygons were used as changed sampling area. The model prediction result, which is presented in the form of binary mask, was post-processed by filling the holes of the binary mask, since it assumes that isolated unaffected area should not exist in the face of such severe natural disaster.

Figure 6a shows a case study area for Bahamas hurricane Dorian 2019. Figure $6 \mathrm{~b}$ shows the impacted area as computed by change detection method. The impacted area image indicates that the hurricane Dorian was so intense that it impacted all the land features in the study area, covering both vegetation and infrastructure areas. The pre-event building footprints were overlaid onto the impacted area map to derive the footprints of impacted buildings. Figure $6 \mathrm{c}$ zooms in two subareas with pre- and post-event images, together with impacted building footprints. It shows that all the buildings did suffer from the Dorian, with some of them receiving major damage and others being totally destroyed.

\section{M5: ACCELERATED KNOWLEDGE FROM GROUND-LEVEL IMAGES}

In this section we present our work on extracting complex and qualitative information from disaster related images (particularly from ground imagery of disaster incidents from social media). This also serves as a second line of evidence for the damages. Extracting complex/ qualitative/ subjective information from ground imagery is much more challenging than extracting quantitative/ objective/ directly conclusive information (e.g., people counting, identifying animals, detecting popular places or structures). In this work we have identified the following disaster related information classes as the set of complex and overall qualitative information to extract from images:

- “1_Rescue_groups”: image showing smaller scale rescue groups and volunteers in action (smaller than government, military or large rescue organizations).

- "2_Rescue_military_or_larger_organizations": image showing larger scale rescue organizations in action for rescue effort. 


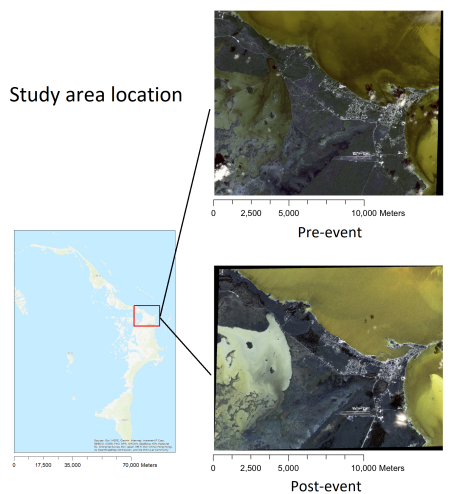

(a) Case study area for satellite image based change detection for Bahamas hurricane Dorian 2019.

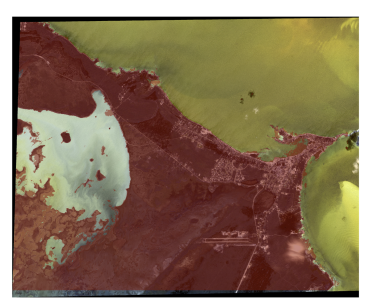

(b) Map of impacted area (red color). a. pre-event

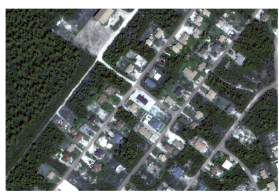

d. pre-event

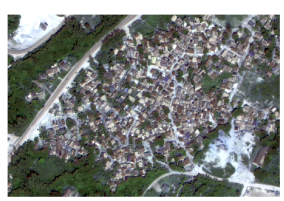

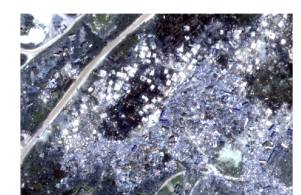

b. post-event

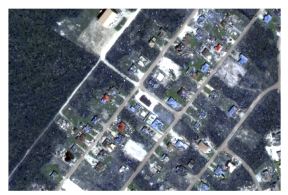

e. post-event

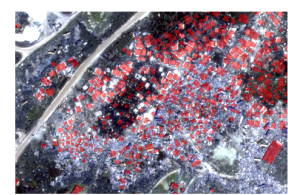

c. impacted buildings

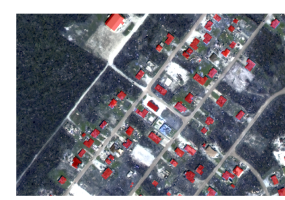

f. impacted buildings (c) Zoomed-in areas of impacted buildings.

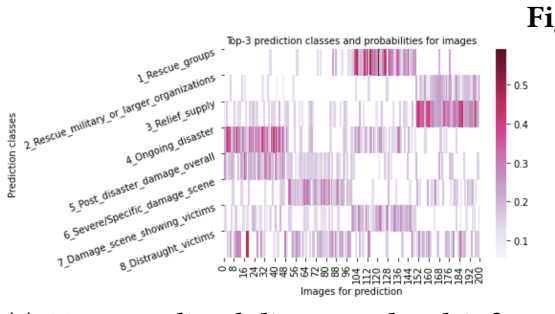

(a) Top-3 predicted disaster related information classes (along with corresponding prediction probabilities) for images mation classes (along with correspond- of the distribution is shown for two different types of of event Bahamas hurricane Dorian from Aug-Sep 2019.

Figure 6: Change detection from satellite imagery.

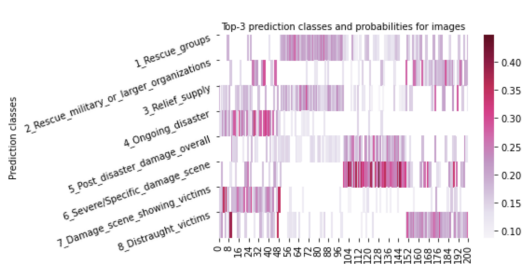

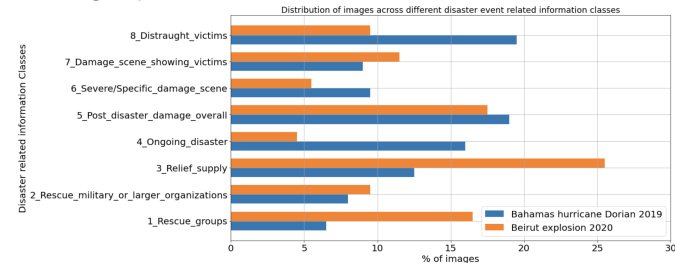

(c) Distribution (\%) of disaster event images across varing prediction probabilities) for images of disaster events: Baham
event Beirut explosion from Aug 2020.

Figure 7: Accelerated and fine-grained knowledge extraction from ground-level images.

- "3_Relief_supply": image showing various items of relief supply being distributed or delivered.

- "4_Ongoing_disaster": image of ongoing disaster incident.

- “5_Post_disaster_damage_overall”: image of post-disaster damages, but showing overall high-level damage situation of the areas affected.

- “6_Severe/Specific_damage_scene”: image of post-disaster damages, but focusing on or highlighting specific damage scenarios.

- "7_Damage_scene_showing_victims": image showing affected victims inside the damage or disaster scene.

- "8_Distraught_victims": image showing distraught or affected victims, but not in the backdrop of disaster scene.

These information classes are also illustrated in the Appendix (Section A.4 Figure 12) with example ground images from two different disaster incidents of two different nature (Bahamas hurricane Dorian 2019 and Beirut explosion 2020). To note that this selection of complex information classes can vary through applications and changing interests of information extraction from images. It can also vary with point of view of disaster incident analytics. Now we present our designed algorithm approach for accelerated extraction of these information classes from ground images.

- Algorithm approach. Our designed system has two goals: (i) training quickly for accelerated classification model design and deployment; (ii) training from very few labeled images (ground truth data) for rapid deployment with changing focus (information classes of interest). We have designed our proprietary machine learning based algorithm to extract such complex information from disaster related ground imagery. Compared to more traditional image processing and analytics algorithms, the technical contribution and operational efficiency of our algorithm are as follows: (i) Our solution can learn to extract the focused information classes from very few labeled training data samples (by using few-shot metalearning based deep learning approach $[16,28]$ ); (ii) With these few labeled samples (i.e., ground truth data), our solution can very quickly optimize the prediction model (by only having to train final stage light-weight linear classifier, when majority of pre-training is done in advance for first-stage nonlinear model design, which is also known as embedding).

But more particularly, novelty of our algorithm over state-of-theart recent few-shot meta-learning algorithm in [28] are as follows. Instead of being dependent on single embedding regarding pretraining, we have designed a library of multiple embeddings. These embeddings are pretrained from a completely separate data source, the Placepedia dataset [14] regarding geospatial ground-level images of places or planet-wide points of interests (POI). The classes for pretraining are also completely different from the information classes of interest in this current application. But overall we used 
pretraining dataset and pretraining classes that is still in context with geospatial application and ground-level imagery. We have generated multiple (four) embeddings from the following multiple (four) classification problems in pretraining: (i) identifying popular places (408 classes in the classification problem); (ii) identifying geographic region (250 classes); (iii) detecting district name (183 classes); and (iv) detecting the continent (7 classes).

Using these four above-mentioned pre-training embeddings, we have mapped each image file from our current application of interest to four corresponding non-linear computation of feature vectors. These pretrained embeddings are four separately trained Resnet12 [13] based deep network architecture with convolutional layers and residual connections. Now after these pretraining of embeddings, a separate final stage light-weight linear classifier model is trained/optimized from each of the four feature vectors and the few available labeled ground truth data (dataset available in current application). From the four optimized linear classifier models, the final combination of embedding and linear model is selected that achieves the highest classification accuracy (in this current information classification problem, not the pretraining classification problems). The final generated classification model then becomes the selected embedding (ResNet12 architecture as non-linear model) as the first-stage, followed by the corresponding linear classifier as the second and final stage.

Classification performance results. Example of prediction probabilities of different classes, as computed by our algorithm, is shown in the Appendix (Section A.4 Figure 13), shown for the two different disaster events. Now Figure 7a (Bahamas hurricane disaster) and Figure 7b (Beirut explosion disaster) illustrate top3 selected prediction probabilities and corresponding predicted classes, for each event's example dataset of 200 images. It can be observed (Figure 7a) that the following classes were detected with higher probabilities for Bahamas hurricane disaster event: 1_Rescue_groups, 3_Relief_supply and 4_Ongoing_disaster. But for Beirut explosion disaster event, a little different set of classes were detected with higher probabilities (Figure 7b): 4_Ongoing_disaster, 6_Severe/Specific_damage_scene.

We have made another observation that with changing nature of disaster event and changing geospatial context, the distribution of disaster images across the classes also do vary. Figure $7 \mathrm{c}$ shows the distribution of images across different predicted classes (one final predicted class is selected for each image). Although that distribution will be influenced by the distribution of actually collected image types, due to our collection of images from Twitter hashtag search, the found distribution of image types can be considered a proxy to the actual distribution of image types that was shared and available in social media (during the few days of disaster event and immediate aftermath). It can be observed that there were more images shared/available of 4_Ongoing_disaster and 8_Distraught_victims classes for Bahamas hurricane Dorian 2019 disaster event (compared to that of Beirut explosion 2020 disaster event). But for Beirut disaster event, comparatively more images were shared/available for 1_Rescue_groups and 3_Relief_supply classes. This kind of analytics (when done in accelerated manner) can be beneficial and impactful for remotely observing post-disaster recovery activities, as well as can enable querying the relevant communities

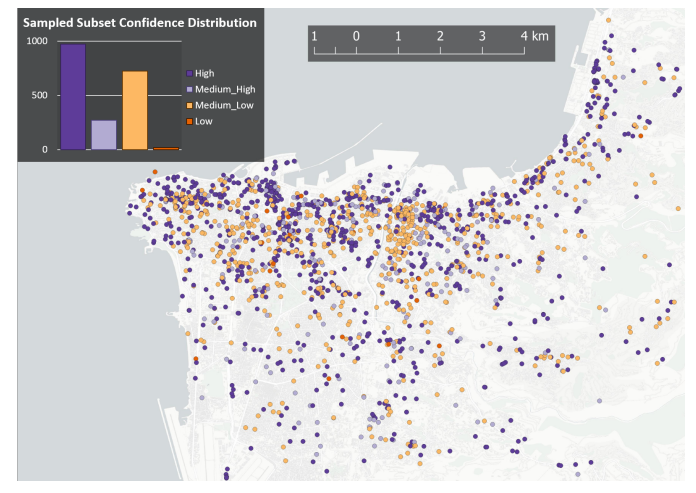

Figure 8: Distribution of confidence values of sampled subset in the QAQC reviewed CIs (for Beirut explosion 2020).

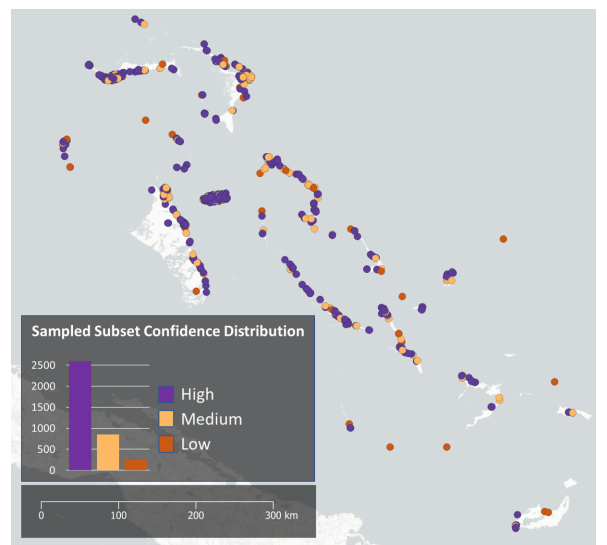

Figure 9: Distribution of confidence values of sampled subset in the QAQC reviewed CIs (for Bahams hurricane 2020, showing Bahamas overall).

or stakeholders further for lesser/missing information types that are shared/available.

\section{M6: FINAL ASSESSMENT AND QUALITY ASSURANCE}

Another important component in our designed system is final assessment with human-in-the-loop based QAQC (quality assurance, quality control). The purpose of the QAQC Process is to add the human factor in determining country and culture specific spatial accuracy and information reliability of reviewed CIs. As millions of CIs are collected from various data sources, the accuracy of the information is not guaranteed due to the inconsistent nature of user-generated data. QAQC is done by our team of geospatial data analysts. The collected and computed geospatial CIs (critical infrastructure) data are reviewed by the QAQC process, with sampling from the overall dataset using a proportional sampling method on places of importance and relevance.

Using satellite imagery and available web-based information sources, CIs are processed to be assigned to and validated with set of possible confidence values: either (a) High, Medium_High, Medium_Low, Low and Not Reviewed (NR); or (b) High, Medium, Low and Not Reviewed (NR). Details of how these confidence values are determined, are explained in the Appendix Section A.5. 
Figure 8 illustrates geospatial locations of the CIs and distribution of their confidence values, in the sampled subset of QAQC for Beirut 2020 explosion event. Similar distribution for the Bahamas 2019 hurricane event is shown in Figure 9.

\section{CONCLUSION}

Accelerated and automated discovery and detection of damages to Critical Infrastructure (CI) immediately after a disaster is key to making a tangible impact on first responders' effort and mitigation measures. This work discussed our experience dealing with the aftermath of Hurricane Dorian in the Bahamas, and the recent warehouse explosion in Beirut, Lebanon. Both destroyed a large swath of infrastructure and impacting human lives. We showed an end-to-end accelerated human-machine teaming workflow on accurately identifying the damaged critical infrastructure with various confidence levels when it's impossible to be physically present at the impacted locations. Specifically, we discussed the development and use of high-throughput data-intensive geospatial architecture, an integrated AI processing for feature extraction, collection, and conflation of disparate spatial data sources, and the irreplaceable importance of using human-in-loop towards improving and accurately estimating the likely damages to CIs in a timely manner. In the future, we would like to expand by creating pre-disaster baseline dataset and templating the workflow as a function of various disaster types, utilizing even more data from IoTs (internet of things), etc. for improving location intelligence.

\section{ACKNOWLEDGMENTS}

This manuscript has been authored by UT-Battelle, LLC, under contract DE-AC05-00OR22725 with the US Department of Energy (DOE). The US government retains and the publisher, by accepting the article for publication, acknowledges that the US government retains a nonexclusive, paid-up, irrevocable, worldwide license to publish or reproduce the published form of this manuscript, or allow others to do so, for US government purposes. DOE will provide public access to these results of federally sponsored research in accordance with the DOE Public Access Plan (http://energy.gov/downloads/doe-public-access-plan).

\section{REFERENCES}

[1] 2000. Measuring Vegetation (NDVI \& EVI) - NASA Earth Observatory

[2] 2011. Public Health Emergency Response Guide for State, Local, and Tribal Public Health Directors. Guideline by the US United States Centers for Disease Control and Prevention (CDC).

[3] 2017. 2017 Hurricane Season FEMA After-Action Report. https: //www.fema.gov/sites/default/files/2020-08/fema_hurricane-season-afteraction-report_2017.pdf.

[4] 2018. FEMA Strategic Plan 2018-2022. https://www.fema.gov/sites/default/files/ 2020-03/fema-strategic-plan_2018-2022.pdf,.

[5] 2021. ORNL LandScan: a community standard for global population distribution data. https://landscan.ornl.gov/.

[6] Ebtehal Turki Alotaibi, Shahad Saleh Alqefari, and Anis Koubaa. 2019. LSAR: Multi-UAV Collaboration for Search and Rescue Missions. IEEE Access 7 (2019), 55817-55832. https://doi.org/10.1109/ACCESS.2019.2912306

[7] G. Bartoli, R. Fantacci, F. Gei, D. Marabissi, and L. Micciullo. 2015. A novel emergency management platform for smart public safety. International fournal of Communication Systems 28, 5 (March 2015), 928-943.

[8] Eric Lucas dos Santos Cabral, Wilkson Ricardo Silva Castro, Davidson Rogério de Medeiros Florentino, Danylo de Araújo Viana, João Florêncio da Costa Junior Ricardo Pires de Souza, Amália Cinthia Meneses Rêgo, Irami Araújo-Filho, and Aldo Cunha Medeiros. 2018. Response time in the emergency services. Systematic review. Acta cirurgica brasileira 33, 12 (2018), 1110-1121.
[9] Charles W Chapman. 2020. Assumption and Adaptation in Emergency Response: Evaluating The Strategic Approach of The National Incident Management System. Technical Report. NAVAL POSTGRADUATE SCHOOL MONTEREY CA.

[10] Omar Cheikhrouhou, Anis Koubaa, and Anis Zarrad. 2020. A cloud based disaster management system. Journal of Sensor and Actuator Networks 9, 1 (Jan. 2020), 6. https://doi.org/10.3390/jsan9010006

[11] Esri. 2020. Create Fishnet-Data Management toolbox | Documentation. https://pro.arcgis.com/en/pro-app/tool-reference/data-management/ create-fishnet.htm

[12] Federal Emergency Management Agency. [n.d.]. OpenFEMA | FEMA.gov.

[13] Kaiming He, X. Zhang, Shaoqing Ren, and Jian Sun. 2016. Deep Residual Learning for Image Recognition. 2016 IEEE Conference on Computer Vision and Pattern Recognition (CVPR) (2016), 770-778.

[14] Huaiyi Huang, Yuqi Zhang, Qingqiu Huang, Zhengkui Guo, Ziwei Liu, and Dahua Lin. 2020. Placepedia: Comprehensive place understanding with multi-faceted annotations. In European Conference on Computer Vision. Springer, 85-103.

[15] Mariam Kiran, Peter Murphy, Inder Monga, Jon Dugan, and Sartaj Singh Baveja. 2015. Lambda architecture for cost-effective batch and speed big data processing. In 2015 IEEE International Conference on Big Data (Big Data). IEEE, 2785-2792.

[16] Kwonjoon Lee, Subhransu Maji, Avinash Ravichandran, and Stefano Soatto. 2019. Meta-learning with differentiable convex optimization. In Proceedings of the IEEE/CVF Conference on Computer Vision and Pattern Recognition. 10657-10665.

[17] Tao Liu, Lexie Yang, and Dalton D Lunga. [n.d.]. Towards Misregistration-Tolerant Change Detection using Deep Learning Techniques with Object-Based Image Analysis. In Proceedings of the 27th ACM SIGSPATIAL International Conference on Advances in Geographic Information Systems. 420-423.

[18] Sergio Luna and Michael J Pennock. 2018. Social media applications and emergency management: A literature review and research agenda. International journal of disaster risk reduction 28 (2018), 565-577.

[19] Nathan Marz and James Warren. 2013. Big Data: Principles and best practices of scalable real-time data systems. Manning.

[20] Ayan Mukhopadhyay, Geoffrey Pettet, Mykel Kochenderfer, and Abhishek Dubey. 2020. Designing Emergency Response Pipelines: Lessons and Challenges. arXiv preprint arXiv:2010.07504 (2020).

[21] OCHA. 2011. ReliefWeb: Informing humanitarians worldwide. http://reliefweb. int/about

[22] Rachel Palumbo, Laura Thompson, and Gautam Thakur. 2019. SONET: A Semantic Ontological Network Graph for Managing Points of Interest Data Heterogeneity. In Proceedings of the 3rd ACM SIGSPATIAL International Workshop on Geospatial Humanities (GeoHumanities âĂŹ19). Association for Computing Machinery, New York, NY, USA, Article Article 6, 6 pages. https: //doi.org/10.1145/3356991.3365474

[23] SE Rigby, TJ Lodge, S Alotaibi, AD Barr, SD Clarke, GS Langdon, and A Tyas. 2020. Preliminary yield estimation of the 2020 Beirut explosion using video footage from social media. Shock Waves 30, 6 (2020), 671-675.

[24] Hansi Senaratne, Amin Mobasheri, Ahmed Loai Ali, Cristina Capineri, and Mordechai (Muki) Haklay. 2017. A review of volunteered geographic information quality assessment methods. International fournal of Geographical Information Science 31, 1 (2017), 139-167. https://doi.org/10.1080/13658816.2016.1189556 arXiv:https://doi.org/10.1080/13658816.2016.1189556

[25] James M Shultz, Duane E Sands, James P Kossin, and Sandro Galea. 2020. Double environmental injusticeâĂŤclimate change, Hurricane Dorian, and the Bahamas. New England fournal of Medicine 382, 1 (2020), 1-3.

[26] Liu Tao and Yang Lexie. 2020(Accepted). A Fully Automatic Method for Rapidly Mapping Impacted Area by Natural Disaster. IEEE, IGARSS 2020 - 2020 IEEE International Geoscience and Remote Sensing Symposium.

[27] Gautam S. Thakur, Budhendra L. Bhaduri, Jesse O. Piburn, Kelly M. Sims, Robert N. Stewart, and Marie L. Urban. 2015. PlanetSense: A Real-Time Streaming and Spatio-Temporal Analytics Platform for Gathering Geo-Spatial Intelligence from Open Source Data. In Proceedings of the 23rd SIGSPATIAL International Conference on Advances in Geographic Information Systems (SIGSPATIAL '15). Association for Computing Machinery, New York, NY, USA, Article 11, 4 pages. https: //doi.org/10.1145/2820783.2820882

[28] Yonglong Tian, Yue Wang, Dilip Krishnan, Joshua B Tenenbaum, and Phillip Isola. 2020. Rethinking few-shot image classification: a good embedding is all you need? arXiv preprint arXiv:2003.11539 (2020).

[29] U.S. National Library of Medicine. [n.d.]. Disaster Apps for Your Digital Go Bag| NLM. https://disasterinfo.nlm.nih.gov/apps

[30] Daniel Wladdimiro, Pablo Gonzalez-Cantergiani, Nicolas Hidalgo, and Erika Rosas. 2017. Disaster management platform to support real-Time analytics. In Proceedings of the $20163 \mathrm{rd}$ International Conference on Information and Communication Technologies for Disaster Management, ICT-DM 2016. Institute of Electrical and Electronics Engineers Inc. https://doi.org/10.1109/ICT-DM.2016.7857207 


\section{A APPENDIX}

\section{A.1 M1: Geospatial Architecture Benchmarks}
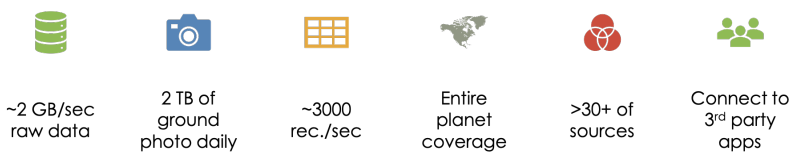

Figure 10: Geospatial architecture performance measures.

Figure-10 shows the benchmarks of geospatial platform proposed Section 2. This data-intensive platform allows for rapid collection and processing of disparate data needed for end-to-end analysis of CIs.

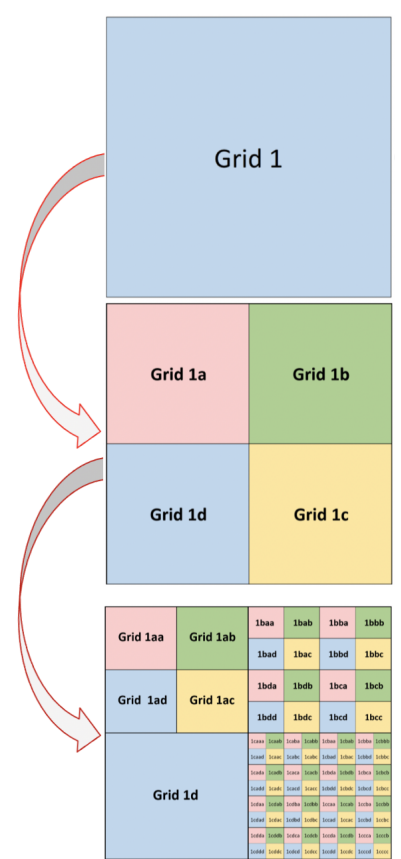

Figure 11: Recursive break-down of large grid in smaller grids as a function of population distribution.

\section{A.2 M2: Dynamically Spaced Geogrid}

This is in continuation from Section 3.2 of main paper content. As shown in Figure-11 From the left quadrant clockwise, we labeled the four quadrants a, b, c, and d, respectively. These quadrant labels were then appended to the original ID of the grid being geo-hashed. The iterations of geo-hashing grids and the product of each new quadrant's ID are illustrated. Grid 1 has a population over 5,000 and thus is replaced with four new grids labeled Grid 1a, Grid 1b, Grid $1 \mathrm{c}$, and Grid 1d. When necessary, these divisions continue through each new quadrant(s) (Grid 1a, 1b, and 1c) constantly replacing the previous grid with new appropriately sized grids. If a grid's summed population is under 5,000 , the iterations end and no additional divisions occur (Grid 1d).

\section{A.3 M3: Data Enrichment: Geographic Feature Characterization}

This is in continuation from Section 4.2 of main paper content. Prior to the decision to utilize the described machine learning approach (the One-vs-All classification model for each category), we evaluated its available options in enhancing its collected data. Manual inspection of points was a natural first suggestion, but it has a severe speed problem. While the accuracy of manual inspection would likely outperform most, if not all of the potential machine learning methods, the manual resources available would never keep pace with the volume of points collected that require examination.

Option for pattern analysis based implementation followed option for manual inspection, but it would entail extreme complexity to perform even basic analytics. The number of categories tracked in our platform and solution is in the thousands, so any analytic would need to consider the multitude of variations in naming conventions for each given category, alongside attempting to account for regional differences in naming conventions. While a pattern analysis method that allows for arbitrary complexity would likely perform extremely well, the effort necessary to create said method may rival manually assessing points. Machine learning was then suggested to allow for an abstraction of the complex analytics necessary to match a name to a category, while keeping both runtime and development time to a minimum. With many popular machine learning styles having been implemented in several languages for convenient use, development would be reduced to implementation and settings refinement i.e., tuning the parameters.

Implementation analysis. With machine learning being set as the course of choice, we examined its catalog of previous research and retrieved a machine learning implementation that had been created as a binary classifier. Using SVM and Naïve Bayes, the classifier determines whether a point name is classifiable as its trained category. SVM processes its training data such that any given name is transformed into a point in $\mathrm{n}$-dimensional space that is given the property of either correct or incorrect. SVM then generates a line in that $\mathrm{n}$-dimensional space to separate that which is correct and that which is incorrect. SVM then uses that line to determine the probability that a provided point is correct or incorrect.

Naïve Bayes processes training data such that some number of features are determined that each have some probability of occurring in a correct or incorrect result. Determination of provided points is performed by calculating the probability of classification against the probability of the various features present in correct data. The results of the SVM and Naïve Bayes applications are then averaged to create a final percentage representing the confidence of the models that a given name is the target category. Confidence greater than or equal to $60 \%$ is interpreted as a point being the category and less than $60 \%$ being not the trained category. With the final product needing to be a choice amongst known categories, a binary implementation alone was insufficient, but its existence allowed for simple conversion to a One-vs-All implementation.

A One-vs-All methodology is a reduction of a complex problem to a collection of binary classifications. In the implementation used in our solution, models for each category with enough training 
data are created using the combination SVM and Naive Bayes implementation. For a given set of target points, each model is applied in series with the final category assigned to each point being determined by a combination of greatest confidence and model accuracy. This methodology allows for relatively swift training, testing, and application to the large volume of data that is collected in our system.

\section{A.4 M5: Accelerated Extraction of Complex Information from Ground-level Disaster Images}

This is in continuation from Section 6 of main paper content. Figure 12 illustrates the different information classes of our interest, that are aimed to be extracted from ground-level disaster images.

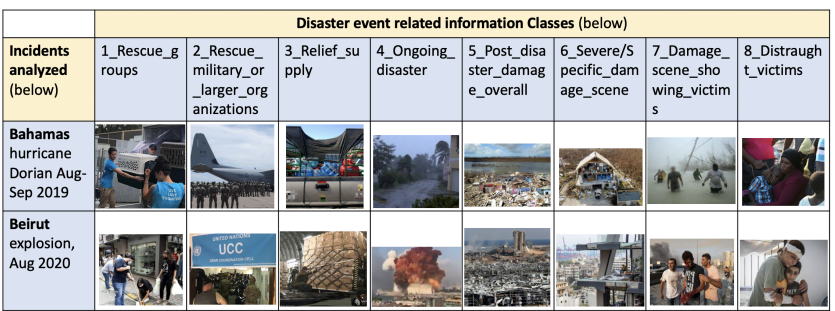

Figure 12: Example of ground imagery i.e., ground photos from social media for each of the disaster related information classes. (shown for two different disaster incidents).

Figure 13 illustrates example prediction output of our algorithm, showing prediction probabilities across different information classes for two example images from two different types of disaster events.

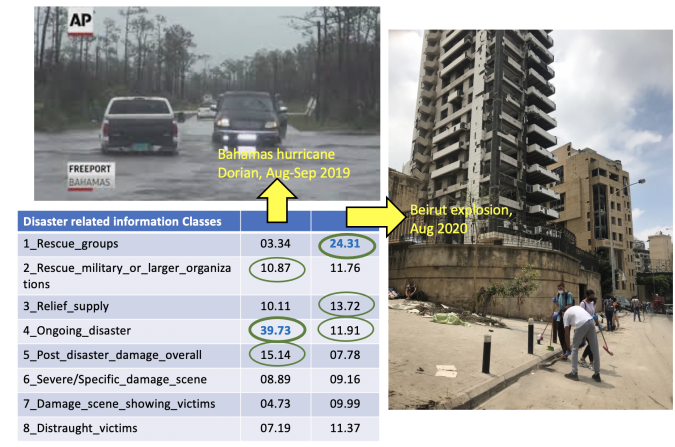

Figure 13: Example of prediction probabilities (\%) for different classes, as predicted by our designed algorithm for disaster related images.

\section{A.5 M6: Human-in-the-loop Quality Assurance}

This is in continuation from Section 7 of main paper content. CIs (Critical Infrastructure) of importance of relevance includes (but are not limited to): healthcare facilities, military facilities, education centers, transportation (airports, ferries, etc.), cultural centers (places of worship, museums, monuments, etc.), sports venues, public buildings (police station, fire station, post office, etc.), communication structures and public utilities.

CIs geospatial datapoints are extracted from satellite imagery and available web-based information sources. Regarding human-in-theloop based QAQC (quality assurance quality control), different confidence values are attributed to the detected geospatially distributed

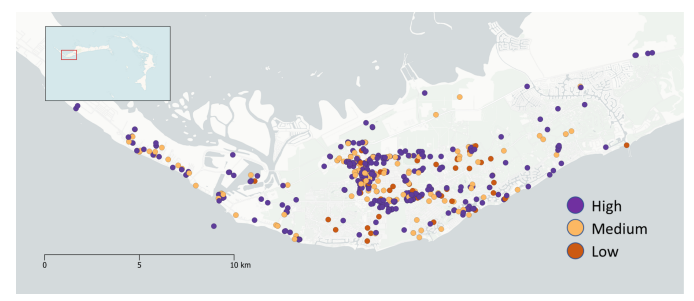

Figure 14: Distribution of confidence values of sampled subset in the QAQC reviewed CIs (for Bahams hurricane 2020, showing Grand Bahama island, a part of overall Bahamas).

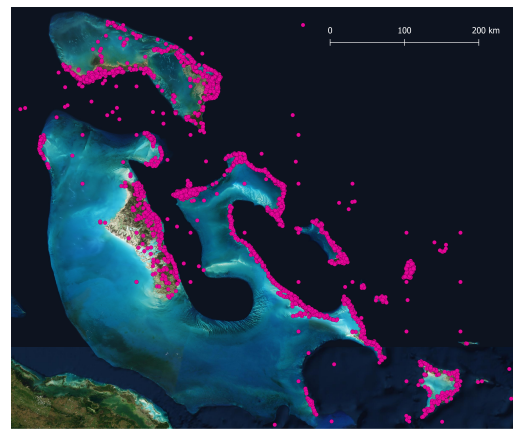

Figure 15: Collected CIs (Bahamas)

CIs (critical infrastructure). High, Medium_High, Medium_Low, Low and Not Reviewed (NR). High CIs data has been verified and is accurate and/or corrected. Medium_High CIs cannot be verified (sourced), but the analyst believes that those CIs' data to be accurate from aerial imagery, intuition, or knowledge of the study area. Medium_Low cannot be verified (sourced) and the analyst cannot be certain of the accuracy of the CIs' data. Low is proven to be inaccurate, yet still the analyst cannot verify a correct location. Not Reviewed CIs were determined to not be of interest by our QAQC (reverted to NULL in deliverable and reports).

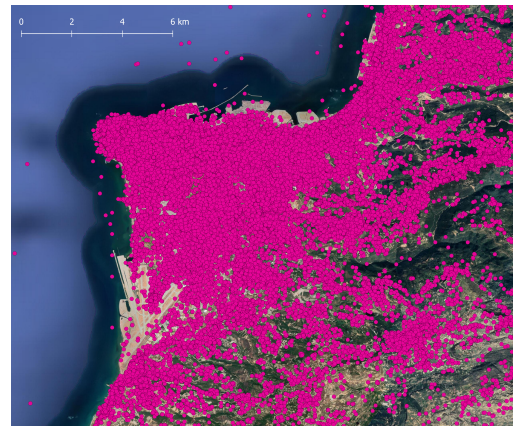

Figure 16: Collected CIs (Beirut)

Regarding the Bahamas hurricane Dorian 2020 disaster event, distribution of the CIs confidence values for overall Bahamas were illustrated in the main section in Figure 9. In this appendix in Figure 14 we have also illustrated geospatial distribution of extracted CIs and their confidence values, specifically for the Grand Bahama island (part of Bahamas). Also Figure 15 and Figure 16 illustrate the collected CIs (critical infrastructure) for Bahamas and Beirut respectively. 